\title{
Pattern Recognition for Region Identification and Labeling for Remotely Sensed Images
}

\author{
Survashe Supriya S \\ Department of Computer Engineering, \\ SVERI's College of Engineering, \\ Pandharpur, India
}

\author{
Korke Ashok G \\ Department of Computer Engineering, \\ SVERI's College of Engineering, \\ Pandharpur, India
}

\begin{abstract}
In today's world, rapid urbanization is leading to increasing the availability of remotely sensed images which gives occasion of identifying urban objects. Lot of work has already done in this regard but it is limited for the images of some specific areas like urban images, airports etc. Also the techniques used were limited to those areas and not applicable to other images from different areas. This paper gives a new method of recognizing the objects of remotely sensed images and labeling them by using classification techniques on the base of knowledge of known images. This system gives identification of the objects in the image and gives labels to the specific area.
\end{abstract}

\section{Keywords}

Remote sensing, Recognition, Pattern recognition, Knowledge-base, Gaussian algorithm

\section{INTRODUCTION}

In machine learning, pattern recognition is the assignment of a label to a given input value. In1936, the discriminate analysis was introduced for this purpose. Examples of pattern recognition include classification that gives challenges for assigning each input value to one of the given set of classes. Here, pattern recognition is a more general problem that encompasses other types of outputs as well.

Pattern recognition algorithms are usually intended to provide a reasonable answer for all possible inputs and to perform "most likely" matching of the inputs after checking their statistical variation. But pattern matching algorithms are used to look for exact matches in the input with patterns which already exist.

In recent years, many different methods of object recognition have applied. But these were limited to some specific area of the certain images for example building, water, bridge, aircraft etc. [1] [4] [5] [6]. The technique is unable to give the preferred output due to change in specific area.

This paper gives advantage of using knowledge of known images as base so that it can work on any type of images like rural and urban. Also, classification technique with edge detection applied for identifying the region under specific category. By labeling them, it gives added advantage of finding specific region percentage to other regions of the remotely sensed images.

\section{PROPOSED TECHNIQUE}

The flow chart of the proposed system is given in Figure 1.

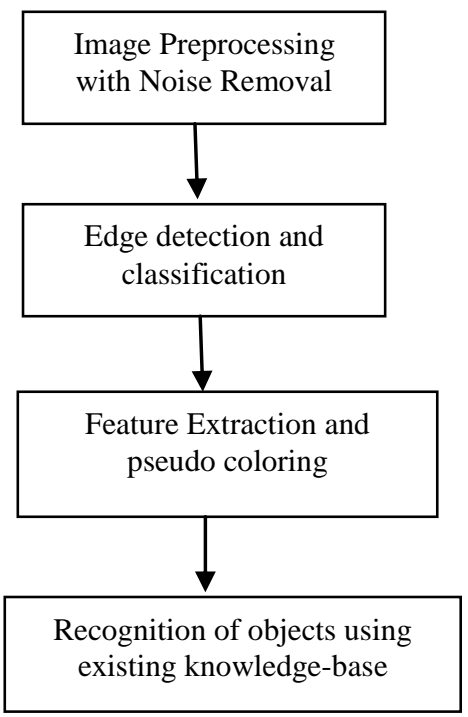

Fig. 1. Flow chart of the proposed technique

The complete process of recognition of object in remotely sensed scene is:

The input image is first given for image pre-processing which transforms the original image into gray level image. Due to this image become clearer for further operation by removing noise and enhancing contrast [4].

Then edge detection algorithms are applied on this noise free image which leads to better classification of one object from other. For this classified image pseudo colors are applied as per the choice for lawn, building, road, water, ground, etc. The part which is not coming under any of this region is considered as unknown if it is not present in knowledge-base. Thus, objects are recognized by pseudo coloring and application of same.

\section{CLASSIFICATION}

\subsection{Image Pre-processing}

Image pre-processing includes correction of deformation that is bending in the image, degradation means filth effects, and noise introduced during the imaging process. This noise may be any unwanted information that pollute an image

This process gives output as a corrected image which is close by both geometrically and radio-metrically near the radiant energy characteristics of the original scene. The errors include radiometric and geometric errors encountered in remotely sensed imagery.

As the study on result of filter algorithms gives Gaussian algorithm is best suited, the following technique is used for 
the image pre-processing. The equation of a Gaussian function in one dimension is

$$
G(x)=\frac{1}{\sqrt{2 \pi \sigma^{2}}} e^{-\frac{x^{2}}{2 \sigma^{2}}}
$$

The product of two such Gaussian functions is considered for two dimensions.

$$
G(x)=\frac{1}{\sqrt{2 \pi \sigma^{2}}} e^{-\frac{x^{2}+y^{2}}{2 \sigma^{2}}}
$$

\subsection{Edge Detection}

This process goes through following steps:

\subsubsection{Applying Gaussian Filter to Remove the Noise and Smooth the Image}

To smooth image, a Gaussian filter is applied to analysis which remove the unevenness of image at this step. Therefore, it will not be significantly affected by separate obvious noise in the image. The equation for size of $2 \mathrm{k}+1 * 2 \mathrm{k}+1$ for a Gaussian filter kernel is shown below:

$$
\mathrm{H}_{\mathrm{ij}}=\frac{1}{2 \pi \sigma^{2}} * \exp \left(-\frac{(\mathrm{i}-\mathrm{k}-1)^{2}+(\mathrm{j}-\mathrm{k}-1)^{2}}{2 \sigma^{2}}\right)
$$

Here is the example of creation of the image to the right, with $\sigma=1.4$ applied on a $5 \times 5$ Gaussian filter. (The convolution operation is denoted by asterisk).

$$
\mathrm{B}=\frac{1}{159}\left[\begin{array}{ccccc}
2 & 4 & 5 & 4 & 2 \\
4 & 9 & 12 & 9 & 4 \\
5 & 12 & 15 & 12 & 5 \\
4 & 9 & 12 & 9 & 4 \\
2 & 4 & 5 & 4 & 2
\end{array}\right] * \mathrm{~A}
$$

\subsubsection{Calculation of Intensity Gradients for the}

\section{Image}

An edge in an image is calculated by Canny edge detection algorithm using four filters on different directions namely horizontal, vertical and diagonal edges in the blurred image. The edge detection operator must (Roberts, Prewitt, Sobel for example) return to a value for the first derivative in the horizontal direction $(\mathrm{Gx})$ and the vertical direction (Gy). From this the edge gradient and direction can be determined:

$$
\begin{aligned}
G=\sqrt{G_{x^{2}}+G_{y^{2}}} \\
\theta=\operatorname{atan} 2(G y, G x)
\end{aligned}
$$

where $\mathrm{G}$ and $\theta$ were calculated by using the above equations.

\subsubsection{Apply Non-Maximum Suppression to}

Throw Out False Responses to Edge Detection.

The result of gradient calculation with the edge extracted from the gradient value is still quite unclear and distorted. This suppression of non-maxima can help to suppress all the gradient values to 0 excluding the local maximal that is indicating the location with the sharpest change of intensity value. The following algorithm is applied for the calculation of each pixel in the gradient image:

1. Compare to the current pixel's edge strength with the edge strength of the pixel in the positive and negative gradient directions.

2. If the edge strength of the current pixel is the largest compared to the other pixels in the mask with the same direction, then the value will be saved. For example the pixel pointing in the y direction will be compared to the pixel above and below it in the vertical axis and preserved. Otherwise, the value will be suppressed.

\subsubsection{Detection of Potential Edge Determination by Applying Double Threshold}

The application of non-maximum compression results in displaying edge pixels is quite accurate to represent the real edge. But there are still some edge pixels were found which formed by noise and color variation may cause trouble in next process. For removal of the fake responses, filtration of the edge pixel with the low gradient value and preservation of the edge with the high gradient value is essential. Thus two different threshold values are calculated as high threshold value and low threshold value. For the strong edge pixels, value of edge pixel's gradient is higher than high threshold value. The pixels are marked as weak edge pixels which value is in between the high threshold value and low threshold value. Those pixels having value smaller than the low threshold value will be suppressed.

\subsubsection{Finalize the Detection of Edge by}

Suppressing all the other Edges that are Weak and Not Connected to Strong Edges.

All these steps tell us that strong edge pixels are involved in the final edge image because they are taken from true edges in the image. So to achieve accurate result, the weak edges must be disposed off. Most of the times it is found that weak edge pixels caused from true edges are connected to strong edge pixels. So Binary Large Object-analysis is used to get these edge connections. It checks weak edge pixel and its 8 neighborhood pixels. As one strong pixel is involved in every BLOB, accordingly weak edge point is identified and preserved.

\section{FEATURE EXTRACTION}

The proposed method associates each region of an image to our database. When user selects his required region of interest, the clicked pixel and its neighboring 10 by 10 pixels are selected. As per the classification of classes, the selected region will be identified and according the names will be given.

Here, database has created on the study of images of those areas which are already known and it gives maximum correct results. By matching these values with the database values the result is calculated.

\section{RESULTS}

The result of the Image Pre-processing with Noise Removal is shown in Figure 2

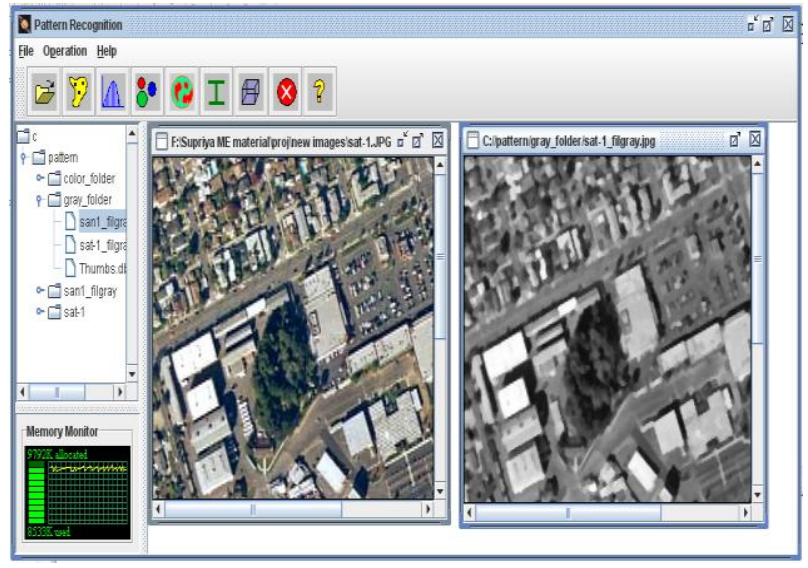

Fig. 2. Image Pre-processing with Noise Removal 
The result of classification is shown in Figure 3.

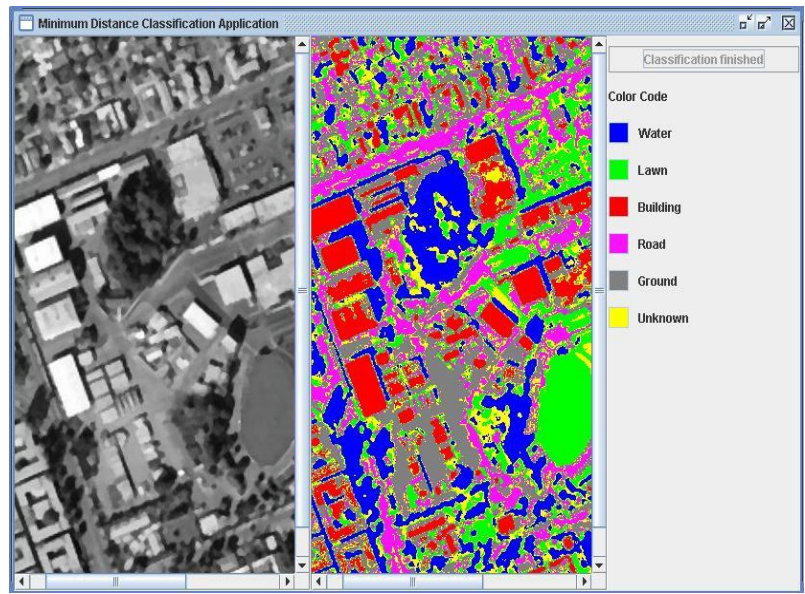

Fig. 3. Images after classification by pseudo coloring

The result of region labeling is shown in Figure 4.

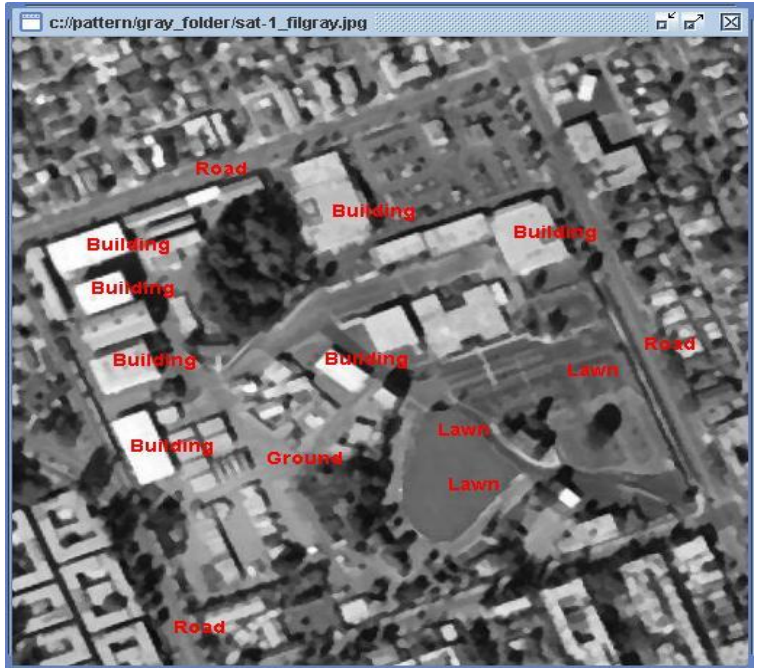

Fig. 4. Image identification and labeling

The figure 5 given below shows the percentage area of all the identified areas.

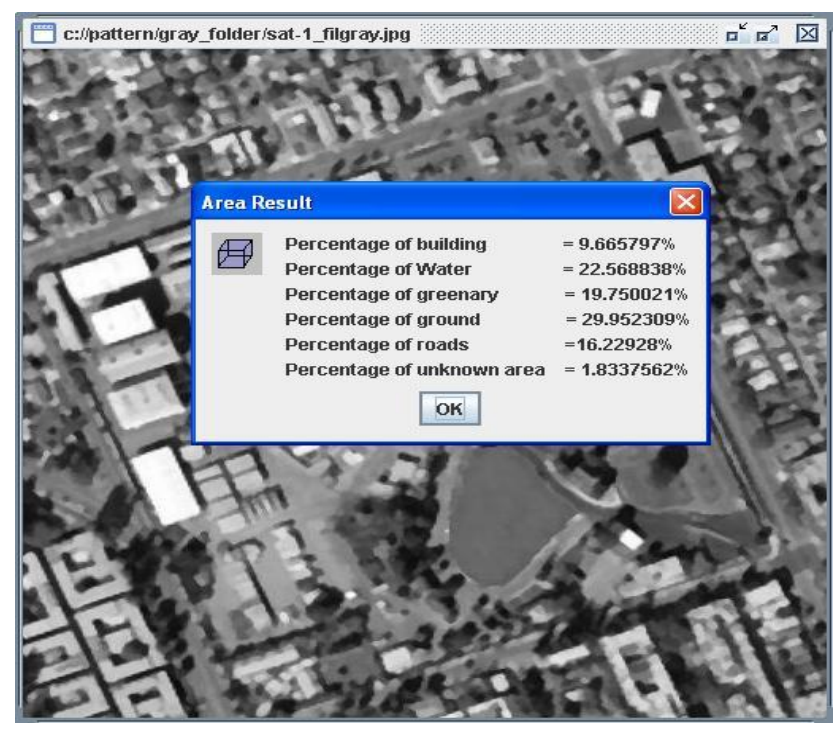

Fig. 5 calculation of percentage area under specific region

\section{CONCLUSION}

This paper gives a new method to identify and label the regions of the remotely sensed images using classification. The results are more efficient and applicable on any images. By applying this technique, the analysis is done on 20 images and results show around $92 \%$ accuracy. This method has been used for the machine learning so any input image can give proper result. This method is used to find the percentage area under civilization, water, and greenery.

In the future, the analysis will be done on more complicated objects. As this system works mainly on color part of the image, other classes like texture, shape can also be used for detecting the objects.

\section{REFERENCES}

[1] G. Forestier, A. Puissant, C. Wemmert, P. Gançarski, "Knowledge-based region labeling for remote sensing image interpretation", Computers, Environment and Urban Systems 36 (2012) 470-480

[2] Jordan Tremblay-Gosselin, Ana-Maria Cretu, "A Supervised Training and Learning Method forBuilding Identification in Remotely Sensed Imaging”, 978-14673-2939-2/13 @2013 IEEE

[3] Nikhil Mantrawadi, Mais Nijim, Young Lee, "Object identification and classification in a high resolution satellite data usingdata mining techniques for knowledge extraction", 978-1-4673-3108-1/13 @2013 IEEE

[4] Jingfei Zhang, Guangmin Sun, "Recognition of Bridge over Water in Remote Sensing Image Using Discrete Hopfield NeuralNetwork", 978-1-4577-1701-7/11 (C)2011 IEEE

[5] Xueyun Chen, Shiming Xiang, Cheng-Lin Liu, and Chun-Hong Pan, "Vehicle Detection in Satellite Images by HybridDeep Convolutional Neural Networks", IEEE Geoscience andRemote Sensing Letters, Vol. 11, No. 10, October 2014

[6] Yiliang Zeng, Jinhui Lan, Chuanzhao Han, Kewei Huang, Jiehui Li, Xuefei Shi, "Aircraft recognition based on improved iterative threshold selectionand skeleton Zernike moment", Optik 125 (2014) 3733-37377.

[7] Susan Niebergall, Alexander Loew and Wolfram Mauser, "Object-Oriented Analysis of Very HighResolution QuickBird Data for Mega City Research inDelhi/India”, 1-4244-0712-5/07 @2007 IEEE

[8] Giorgio Giacinto, Fabio Roli, Lorenzo Bruzzone, "Combination of neural and statistical algorithms forsupervised classi ${ }^{\circledR}$ cation of remote-sensing images", Pattern Recognition Letters 21 (2000) 385-397

[9] M. Fauvel , J.Chanussot, J.A.Benediktsson, "A spatialspectral kernel-based approach for the classification of remote-sensing images", Pattern Recognition 45 (2012) 381-392

[10] Paula Beatriz Cerqueira Leite, Raul Queiroz Feitosa, Antônio Roberto Formaggio,Gilson Alexandre Ostwald Pedro da Costa, Kian Pakzad, Ieda Del'Arco Sanches, "Hidden Markov Models for crop recognition in remote sensing image sequences", Pattern Recognition Letters 32 (2011) 19-26

[11] Mr. Salem Saleh Al-amri, Dr. N.V. Kalyankar, Dr. Khamitkar S.D., "A Comparative Study of Removal Noise from Remote Sensing Image", IJCSI International Journal of Computer Science Issues, Vol. 7, Issue. 1, No. 1, January 2010. 